\title{
What is the true nature of blinkers?
}

\author{
S. Subramanian ${ }^{1}$, M. S. Madjarska ${ }^{1}$, J. G. Doyle ${ }^{1}$, and D. Bewsher ${ }^{2}$ \\ 1 Armagh Observatory, College Hill, Armagh BT61 9DG, N. Ireland, UK \\ e-mail: madj@arm.ac.uk \\ 2 Jeremiah Horrocks Institute, University of Central Lancashire, Preston, Lancashire, PR1 2HE, UK
}

Received 12 August 2011 / Accepted 5 November 2011

\begin{abstract}
Aims. The aim of this work is to identify the true nature of the transient EUV brightenings, called blinkers.

Methods. Co-spatial and co-temporal multi-instrument data, including imaging (EUVI/STEREO, XRT and SOT/Hinode), spectroscopic (CDS/SoHO and EIS/Hinode) and magnetogram (SOT/Hinode) data, of an isolated equatorial coronal hole were used. An automatic program for identifying transient brightenings in CDS O v $629 \AA$ A, EUVI $171 \AA$ and XRT was applied.

Results. We identified 28 blinker groups in the CDS O v $629 \AA$ Aaster images. All CDS O v $629 \AA$ A blinkers showed counterparts in EUVI $171 \AA$ and $304 \AA$ images. We classified these blinkers into two categories, one associated with coronal counterparts and other with no coronal counterparts as seen in XRT images and EIS Fe XII $195.12 \AA$ raster images. Around two-thirds of the blinkers show coronal counterparts and correspond to various events like EUV/X-ray jets, brightenings in coronal bright points or foot-point brightenings of larger loops. These brightenings occur repetitively and have a lifetime of around $40 \mathrm{~min}$ at transition region temperatures. The remaining blinker groups with no coronal counterpart in XRT and EIS Fe XII $195.12 \AA$ appear as point-like brightenings and have chromospheric/transition region origin. They take place only once and have a lifetime of around $20 \mathrm{~min}$. In general, lifetimes of blinkers are different at different wavelengths, i.e. different temperatures, decreasing from the chromosphere to the corona.

Conclusions. This work shows that the term blinker covers a range of phenomena. Blinkers are the EUV response of various transient events originating at coronal, transition region and chromospheric heights. Hence, events associated with blinkers contribute to the formation and maintenance of the temperature gradient in the transition region and the corona.
\end{abstract}

Key words. Sun: atmosphere - Sun: corona - methods: observational - methods: data analysis - sun: transition region

\section{Introduction}

The quasi-steady mechanism(s) that sustains the heating of the outer solar atmosphere and the solar wind is currently under intensive investigation using state-of-the-art ground- and spacebased instrumentation. These observations show that small-scale salt and pepper like bipolar network magnetic fields constantly reconfigure themselves on time scales of minutes-to-hours, resulting in a complicated and dynamically evolving solar atmosphere. It is believed that magnetic reconnection and subsequent energy release is the major mechanism which can relax these constantly evolving magnetic field structures. Such smallscale energy releases seen as sudden brightenings (blinkers or extreme-ultraviolet (EUV) brightenings), fast plasma ejections (spicules or EUV/X-ray jets), etc., are omnipresent and have been reported at different heights in the solar atmosphere. It is crucial to establish links between these different events as they potentially connect the lower and upper solar atmosphere and, hence, could contribute to the transfer of mass and energy in the atmosphere (Parker 1988; Schrijver et al. 1997, 1998; Moore et al. 1999; Winebarger et al. 2002; Yamauchi et al. 2005).

EUV brightenings, also called blinkers, were first reported by Harrison (1997) in the quiet Sun, at transition region (TR) temperatures, using the Coronal Diagnostic Spectrometer (CDS; Harrison et al. 1995) onboard SoHO spacecraft. They show intensity enhancements of a factor of 2-3 (Harrison 1997; Bewsher et al. 2002) and Doppler velocities of $25-30 \mathrm{~km} \mathrm{~s}^{-1}$ (Bewsher et al. 2003) in TR lines like $O$ v $629.77 \AA\left(T \approx 2.4 \times 10^{5} \mathrm{~K}\right)$. The average lifetime of these EUV brightenings is $16 \mathrm{~min}$ ranging from 6 to $40 \mathrm{~min}$ (Bewsher et al. 2002). They have also been observed in active regions (Walsh et al. 1997; Parnell et al. 2002; Bewsher et al. 2003), with slightly higher Doppler velocities of 20-40 $\mathrm{km} \mathrm{s}^{-1}$ (Bewsher et al. 2003). Corresponding signatures of blinkers were found in chromospheric lines (Brković et al. 2001; Brković \& Peter 2003; Brooks et al. 2004; Brooks \& Kurokawa 2004). Harrison et al. (1999) derived an average intensity increase of $4 \%$ and $7 \%$ in the coronal $\mathrm{Mg}$ Ix $368.9 \AA$ and $\mathrm{Mg}$ x $624.9 \AA$ lines, respectively, for these events. Bewsher et al. (2002) also detected a weak response of blinkers in these lines and concluded "that blinkers have no coronal signatures", although "it may be simply that these lines are too weak to detect anything in". Priest et al. (2002) suggested from simple physical models that blinkers can be produced by five different physical mechanisms, namely: the heating of cool spicular material; the containment of plasma in low-lying loops in the network; the thermal linking of cool and hot plasma at the feet of coronal loops; the heating and evaporation of chromospheric plasma in response to a coronal heating event; and the cooling and draining of hot coronal plasma when coronal heating is switched off.

Coronal jets are dynamic features which are observed as collimated ejections of plasma on small scales. They were first observed with the solar X-ray telescope onboard the Yohkoh satellite (Shibata et al. 1992) and are believed to be the result of magnetic reconnection (Yokoyama \& Shibata 1995). The X-ray Telescope (XRT; Golub et al. 2007) onboard Hinode opened a new era for studying X-ray features in tens-of-second detail, revealing the association of some jets with the expansion and eruption of coronal bright point loops 

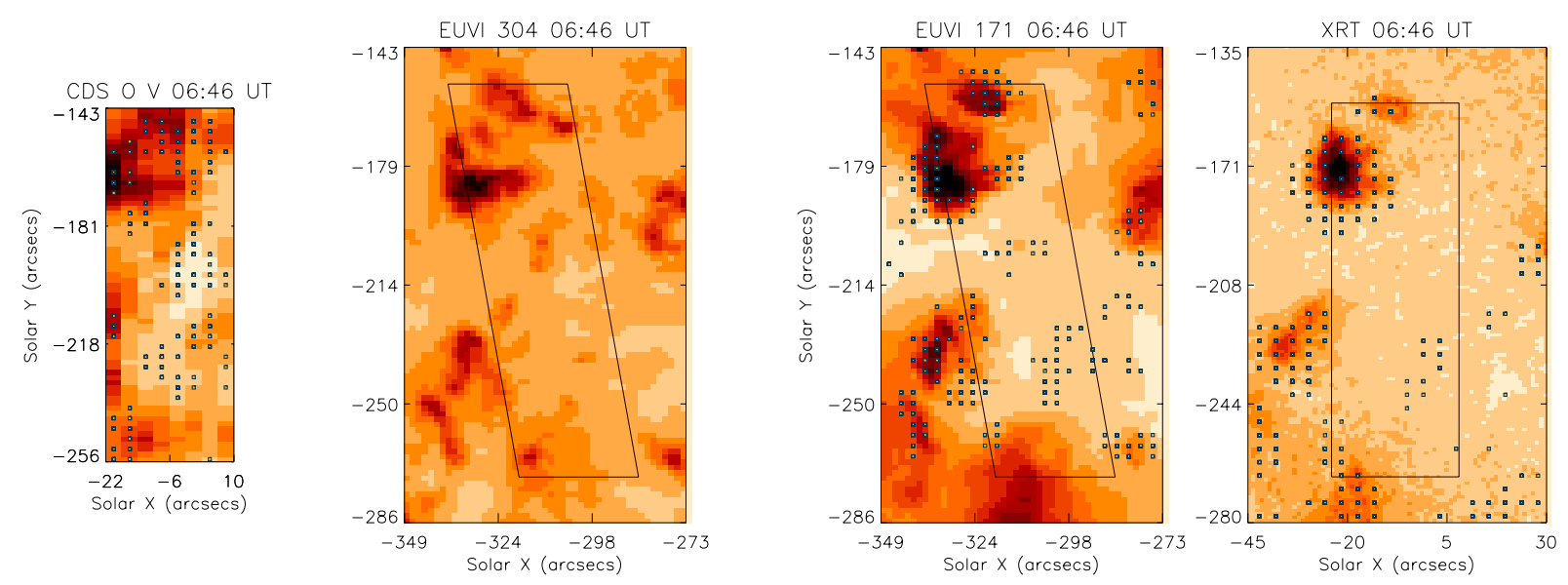

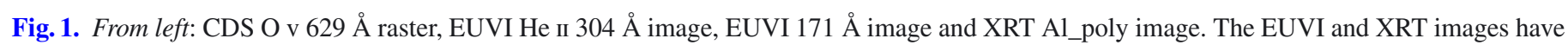
the field of view of the CDS raster over-plotted as a black rectangular box. The identified brightenings in CDS, EUVI and XRT are over-plotted with boxes in the respective images. The difference in the orientation and the coordinates of the EUVI image with respect to the CDS and XRT field-of-views is due to the difference in the viewing angles between STEREO-A and the SoHO and Hinode spacecrafts.

(Shimojo et al. 2007; Filippov et al. 2009; Madjarska 2011). More recently, Subramanian et al. (2010) completed a statistical study of X-ray brightenings in coronal holes and quiet Sun regions. The authors found that more than $70 \%$ of the brightenings observed in coronal holes were coronal jets. However, very few jet-like events were observed in the quiet Sun. The authors also suggested that the remaining unresolved X-ray brightenings could involve two sided loop reconnection (Shibata et al. 1994) between the emerging flux and the overlying coronal field or flows in loop structures perhaps triggered by reconnection shocks from neighbouring regions. For more detailed introduction see Subramanian et al. (2010) and Madjarska (2011).

An intensity increase, i.e. a brightening, either seen in imager or spectrometer data can be a signature of various physical processes. This study aims at investigating the signature of which phenomena the EUV brightenings, often referred to as blinkers, are? We used state-of-art co-observations from imagers and spectrometers which presently observe the Sun with unprecedented spatial and temporal resolution as well as a spectral coverage of a temperature range which provides a reliable coronal diagnostic.

The paper is organized as follow: Sect. 2 describes the observational material. In Sect. 3 we outline the procedure for blinker identification in images taken in spectral lines or imager passbands covering different temperatures, i.e. chromospheric, transition region or coronal. Section 4 presents the obtained results. The discussion and conclusions are given in Sect. 5.

\section{Data reduction and preparation}

Observations obtained with the Solar Optical Telescope (SOT; Tsuneta et al. 2008), the EUV imaging spectrometer (EIS; Culhane et al. 2005, 2007) and XRT all onboard Hinode, the CDS onboard SoHO and the EUV Imager (EUVI; Howard et al. 2008) onboard STEREO were used in the analysis. Approximately $10 \mathrm{~h}$ of data were taken during a dedicated observing run of an isolated equatorial coronal hole (ECH) on 2007 November 12.

X-ray images from XRT/Hinode were taken with the Al_poly filter with a pixel size of $1^{\prime \prime} \times 1$ ", a field-of-view (FOV) of $370^{\prime \prime} \times 370^{\prime \prime}$ and a cadence of $40 \mathrm{~s}$. The data were reduced using xrt_prep.pro which includes despiking, normalisation to data numbers per second to account for variations in exposure times, satellite jitter and orbital variation corrections.

EIS/Hinode was observing in a rastering mode with the $2^{\prime \prime}$ slit. The data consist of a large raster with a FOV of $120^{\prime \prime} \times 496^{\prime \prime}$ followed by 34 small rasters with a FOV of $24^{\prime \prime} \times 248^{\prime \prime}$ taken with a cadence of $12.5 \mathrm{~min}$, and a pixel size of $2^{\prime \prime} \times 1^{\prime \prime}$. Many spectral lines were available for analysis, however, in the present study only the O v $192.9 \AA\left(\log T_{\max }=5.4 \mathrm{~K}\right), \mathrm{Fe}$ vIII $185.21 \AA$ $\left(\log T_{\max }=5.8 \mathrm{~K}\right), \mathrm{Fe} \times 184.59 \AA\left(\log T_{\max }=6.0 \mathrm{~K}\right)$ and Fe XII $195.12 \AA\left(\log T_{\max }=6.1 \mathrm{~K}\right)$ lines are used. The data were reduced using eis_prep.pro and were corrected for EIS detector tilt and satellite orbital variation.

$\mathrm{SOT} /$ Hinode provided both $\mathrm{Ca}$ II $\mathrm{H}$ images and magnetograms. The $\mathrm{Ca}$ II $\mathrm{H}$ images were taken with a pixel size of $0.11^{\prime \prime} \times 0.11^{\prime \prime}$ and a cadence of approximately $90 \mathrm{~s}$. The magnetograms were taken using the Na I $5896 \AA$ filter with a pixel size of $0.16^{\prime \prime} \times 0.16^{\prime \prime}$ and a cadence of $90 \mathrm{~s}$. Both datasets were reduced using $f g \_$prep.pro.

Full disk EUV images from EUVI/STEREO-A were taken using the $171 \AA$ filter (dominated mostly by $\mathrm{Fe} \mathrm{IX} / \mathrm{x}$, but in some instances may have a large contribution from TR lines such as $\mathrm{O} v$ and $\mathrm{O}$ vI, more on this below) with a pixel size of $1.6^{\prime \prime} \times 1.6^{\prime \prime}$ and a cadence of $150 \mathrm{~s}$. The images were reduced with euvi_prep.pro.

CDS/SoHO observed the He r $584.3 \AA$, He II $303.78 \AA$ and $303.786 \AA$ (seen in second order; hereafter $304 \AA$ ) and O v $629.77 \AA$ (hereafter $629 \AA$ ) lines and a spectral window centred around $560 \AA$ containing $\mathrm{Ca} \mathrm{x}$ and $\mathrm{Ne}$ vi lines in rastering mode. Only the O v $629 \AA$ data were used in this paper. The data consist of a large raster with a FOV of $240^{\prime \prime} \times 240^{\prime \prime}$ followed by 40 small rasters with a FOV of $40^{\prime \prime} \times 124^{\prime \prime}$ taken with a cadence of $4 \mathrm{~min}$ and a pixel size of $4^{\prime \prime} \times 1.68^{\prime \prime}$. The data were reduced using the standard software packages for the correction of missing pixels, cosmic ray hits, CCD bias effects and flat-field effects. An intensity map of the emission from the spectral window was obtained by integrating over the spectral line.

The CDS rasters, SOT, EUVI and XRT images were derotated to a common reference time in order to compensate for the solar rotation. Furthermore, the data from the different instruments were co-aligned in order to follow the same region at different temperature regimes. The CDS O v $629 \AA$, the SOT 
S. Subramanian et al.: The nature of blinkers
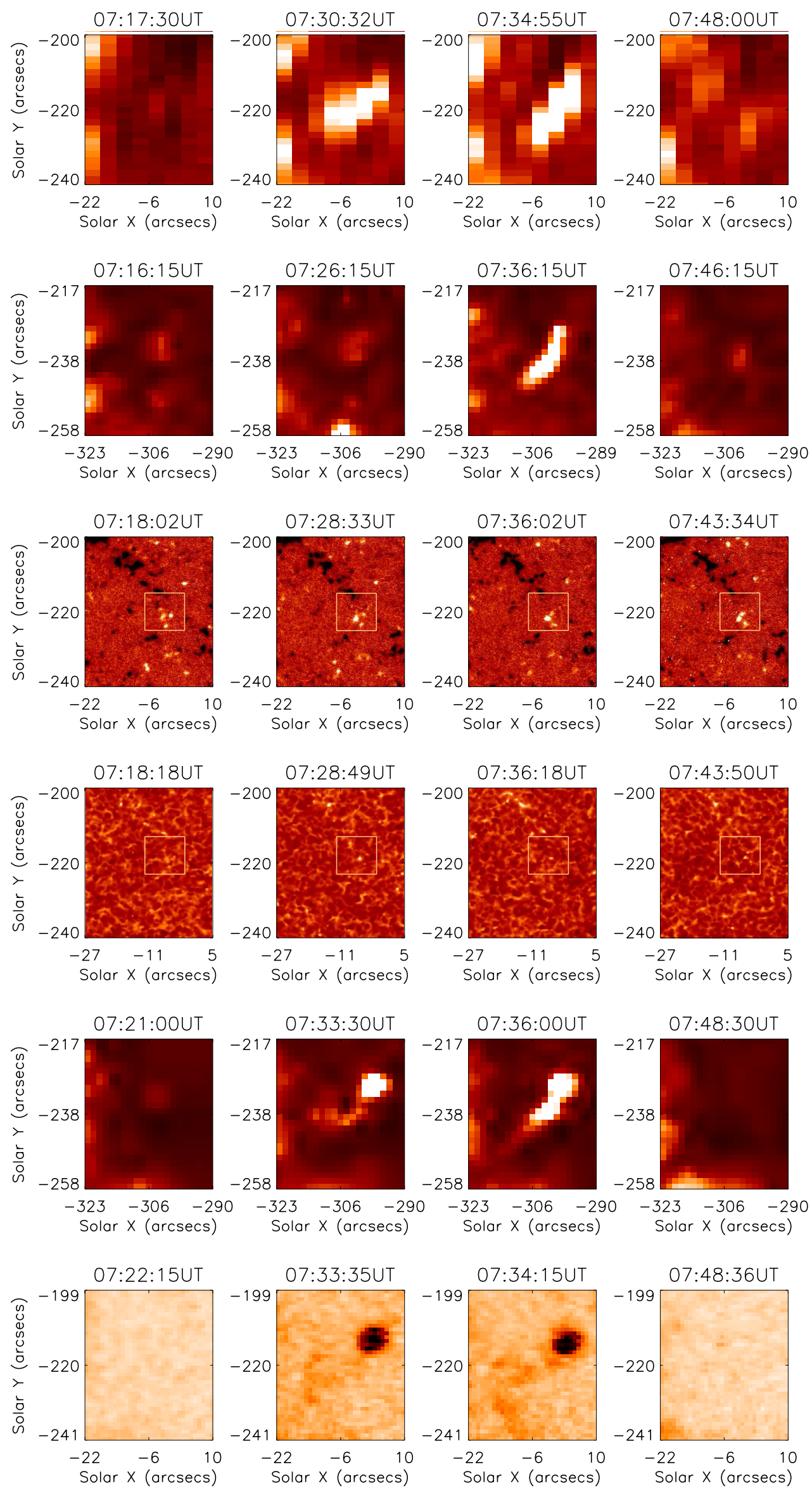

Fig. 2. Blinker group 1 from top in CDS O v $629 \AA$ A, EUVI $304 \AA$, SOT FG magnetograms, SOT Ca II H, EUVI $171 \AA$ and XRT Al_poly. The corresponding magnetogram field-of-view from which the lightcurve of the positive flux was derived, is outlined by the white box on the SOT FG magnetograms and $\mathrm{Ca}$ II $\mathrm{H}$ images. 
$\mathrm{Ca}$ II $\mathrm{H}$ and the magnetogram data were co-aligned with respect to the TRACE $1550 \AA$ images (which were also taken as part of the dedicated observing run, but not used in the scientific analysis presented here). Then the EIS O v $192.90 \AA$ intensity images were co-aligned with the CDS O v $629 \AA$ ones. The EIS Fe XII $195.12 \AA$ data were then cross correlated with the XRT data, which were in turn aligned with the EUVI $171 \AA$ data. The coordinates are unique to the EUVI image (Fig. 1) and cannot be compared with the Hinode and SoHO images due to the difference in the viewing angles between STEREO, Hinode and SoHO. Figure 1 shows a sample (from left) CDS O v $629 \AA$ raster, EUVI He II $304 \AA$ image, EUVI $171 \AA$ image and XRT image with the field of view of the CDS raster over-plotted as a rectangular box.

For the present study, we used data taken of a coronal hole region as they are ideal for this work because of their low background emission at coronal temperatures when compared to the quiet Sun or active region corona. The lightcurves were smoothed with a smoothing window of width 5 for the $\mathrm{Ca}$ II $\mathrm{H}$ and 3 for the rest of the lightcurves. Then the lightcurve values were normalized with respect to the maximum radiance.

\section{Blinker identification}

The first step was to identify brightenings in the CDS O v $629 \AA$ rasters. For this we applied an automated brightening identification procedure described in Subramanian et al. (2010). The procedure finds intensity enhancements in the input light-curves which are above a user defined intensity threshold together with the corresponding start and end times. The input light-curves were made by summing over $1 \times 2$ pixels $^{2}$, which is approximately $4^{\prime \prime} \times 3.4^{\prime \prime}$, and were smoothed over a time window of 3 (i.e. over 3 consecutive images) to remove unwanted spikiness in the background. As CDS was rastering completely inside the coronal hole, with no quiet Sun region, we used a single intensity threshold of $1.45 \times$ the background. The program estimates locally the background for each lightcurve by eliminating the identified intensity peaks and averaging over the rest of the lightcurve. A duration threshold of $45 \mathrm{~min}$ was also used as blinkers can have lifetimes from $6 \mathrm{~min}$ to around $40 \mathrm{~min}$ (Bewsher et al. 2002).

The same procedure was used to identify brightenings in the EUVI $171 \AA$ images and X-ray images with an intensity threshold of $1.35 \times$ and $2 \times$ the background, respectively, and a duration threshold of $45 \mathrm{~min}$ in order to identify the counterparts of the CDS blinkers. The input light-curves were made by summing over $2 \times 2$ pixels $^{2}$, which is approximately $3.2^{\prime \prime} \times 3.2^{\prime \prime}$ for the EUVI $171 \AA$ data and $4 \times 4$ pixels ${ }^{2}$, which is approximately, $4^{\prime \prime} \times 4^{\prime \prime}$ for the X-ray data. The intensity thresholds used in this work were calculated with a trial and error method.

Figure 1 shows sample images with the identified brightenings over-plotted. A total of 96 brightenings were identified in the CDS O v $629 \AA$ raster images. These brightenings were not uniformly distributed over the field of view, instead a number of brightenings occurred close to each other, forming clusters. An event bigger than the pixel scale of one light-curve will have its imprint in adjacent light-curves. Hence, a group of identified brightenings which occurred close together and showed similar light-curves and lifetimes, were visually grouped into individual events. We identified 28 such blinker groups over the course of our observations in the CDS O v $629 \AA$ data. In addition to the automatic procedure, each brightening identified in CDS O v $629 \AA$ was visually cross-correlated with the EUVI

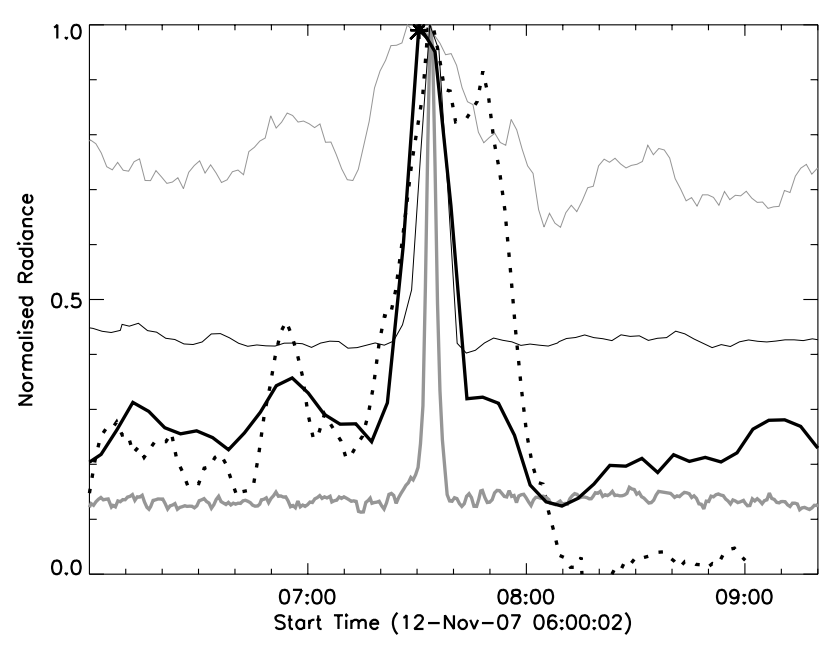

Fig. 3. Light-curves in CDS O v $629 \AA$ (thick black line), EUVI $171 \AA$ (thin black line), SOT Ca II H (thin grey line) and XRT Al_poly (thick grey line) along with the light-curve of the corresponding magnetic fluxes. The light curve of the positive magnetic flux is shown with a dotted line. The asterisk symbol indicates the peak in CDS O v $629 \AA$ A.

$171 \AA$ and X-ray data to search for its counterpart. We found that all CDS O v $629 \AA$ blinkers have a counterpart in the EUVI $171 \AA$ images, while only $57 \%$ of them were present in XRT images. Thus, we classified the identified blinker groups into two categories, one with an X-ray counterpart and the other with no $\mathrm{X}$-ray counterpart.

\section{Results}

\subsection{Blinkers with $X$-ray counterparts}

Out of the 28 identified blinker groups, 16 showed X-ray counterparts in the XRT images with a lifetime of around $40 \mathrm{~min}$ in CDS O v $629 \AA$. These blinkers were further classified into two categories, those associated with pre-existing X-ray coronal bright points and those that were not. A sample of events from each case are discussed below.

The blinker group 1 (BG1) consisting of 15 automatically identified brightenings in CDS O v $629 \AA$ is shown in Fig. 2. This figure also shows the blinker group as seen in EUVI $304 \AA$ (row 2), the corresponding SOT FG magnetograms in $\mathrm{Na}$ I $5896 \AA$ (row 3), SOT Ca II H (row 4), EUVI $171 \AA$ (row 5), and X-ray Al_poly (row 6). Light-curves of BG1 (Fig. 3) in CDS O v $629 \AA$ (thick black line), EUVI $171 \AA$ (thin black line), SOT Ca II H (thin grey line) and XRT Al_poly (thick grey line) along with the light curve of the associated positive magnetic flux (dotted line) from the SOT magnetograms clearly show simultaneous intensity enhancements in all wavelengths during the brightening event. As the EUVI $304 \AA$ dataset has a low temporal resolution of one image every $10 \mathrm{~min}$, we could not use them to follow the structure and dynamics of blinker groups over their lifetime. In Fig. 2, BG1 can be seen as an unresolved brightening with an elongated shape in CDS O v $629 \AA$ and EUVI $304 \AA$ with a lifetime of 45 min in CDS O v $629 \AA$. They show a sudden brightening with a faint ejection of plasma, i.e. a coronal jet, in EUVI $171 \AA$ and XRT Al_poly data with a lifetime of 20 and $16 \mathrm{~min}$, 

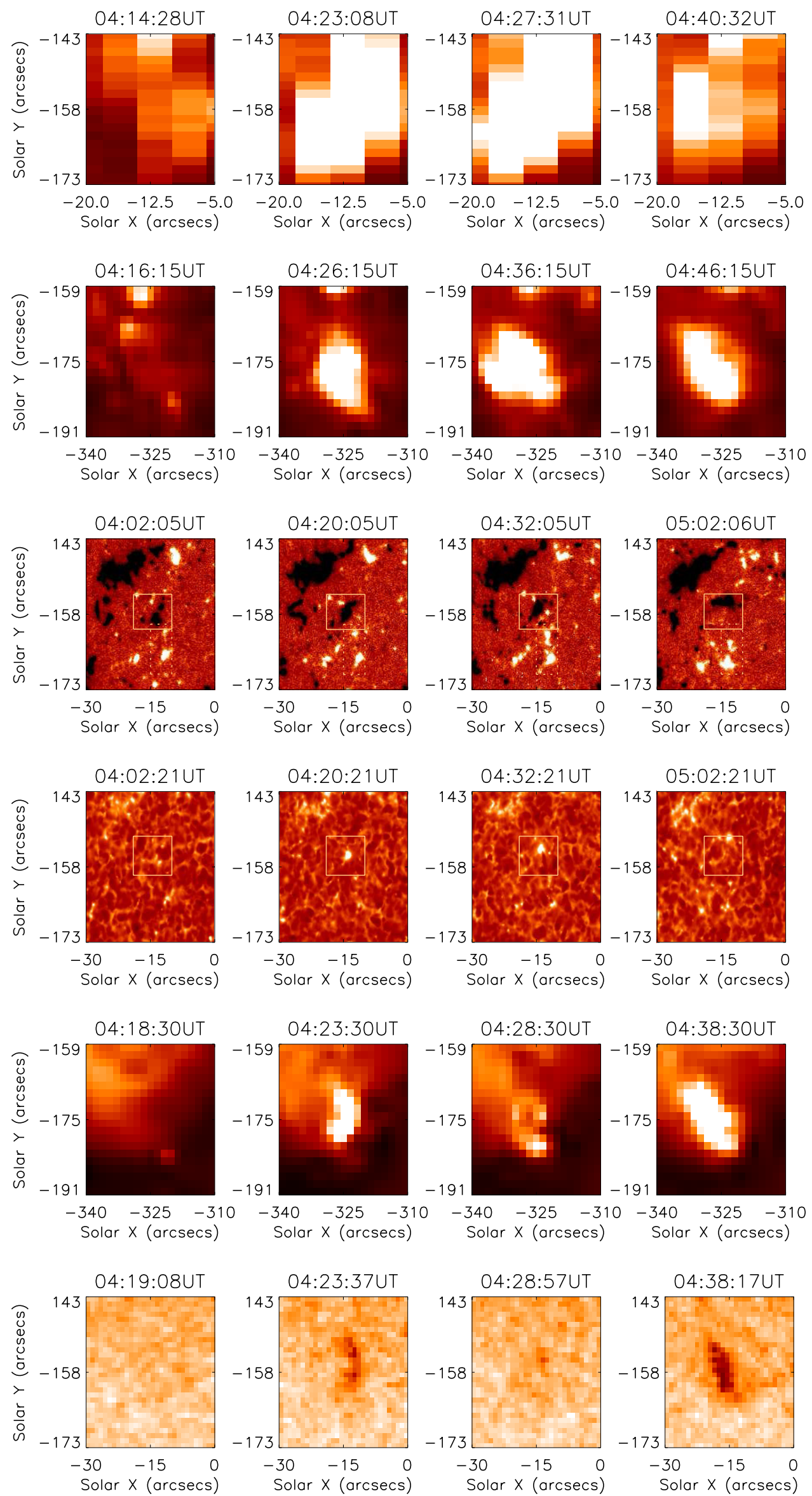

Fig. 4. Blinker group 2 from top in CDS O v 629 Å, EUVI 304 Å, SOT FG magnetograms, SOT Ca II H, EUVI $171 \AA$ and XRT Al_poly. The corresponding magnetogram field-of-view from which the lightcurves of the negative (solid line box) and positive (dashed line) fluxes were derived, is outlined on the SOT FG magnetograms and $\mathrm{Ca}$ II $\mathrm{H}$ images. 


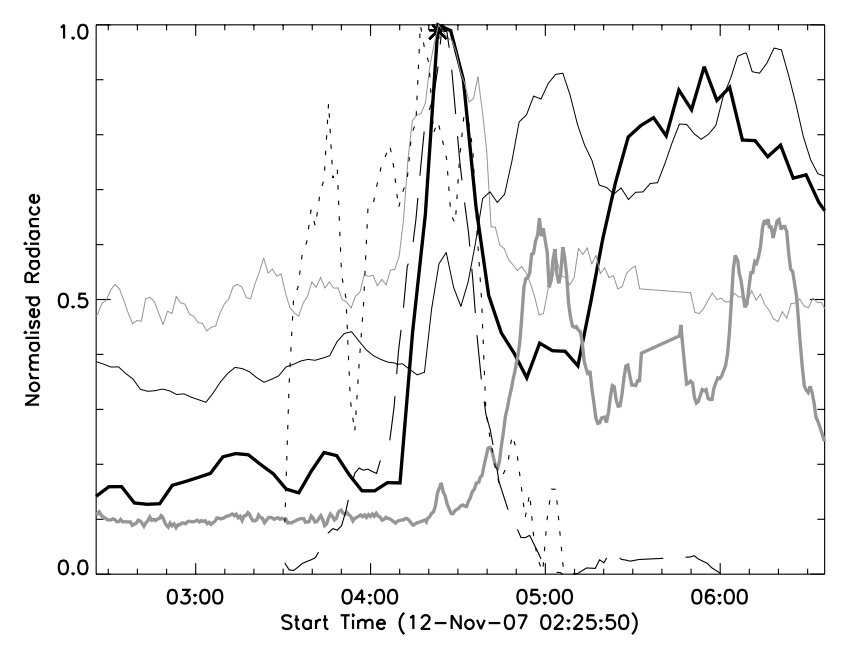

Fig. 5. Light-curves of blinker group 2 in CDS O v $629 \AA$ (thick black line), EUVI $171 \AA$ (thin black line), SOT Ca II H (thin grey line) and XRT Al_poly (thick grey line) along with the light-curve of the corresponding magnetic fluxes. The light curves of the positive and the negative magnetic fluxes in absolute value are shown with dotted and dashed line, respectively. The asterisk symbol represents the peak in CDS O v $629 \AA$.

respectively. It is also observed as a chromospheric brightening in the SOT Ca II H images with a lifetime of $52 \mathrm{~min}$.

The corresponding magnetogram FOV is marked with a white box in Fig. 2, rows 3 and 4. In the magnetograms, we observed a cancellation of weak bipolar fluxes prior to the event and an emergence of a new positive polarity flux during the course of the event. The light-curve of this newly emerged positive polarity (Fig. 3) is very similar to the light-curves of the event in other wavelengths which suggests that the event was triggered by flux emergence. The brightening disappeared when the plasma ejection ceased as seen in the EUVI $171 \AA$ and X-ray images. This blinker/jet falls under the category of an event with no pre-existing features at coronal temperatures, as discussed in Subramanian et al. (2010). This type of jets does not have a repetitive nature and neither do the corresponding blinkers.

Another blinker group, referred to as blinker group 2 (BG2), is discussed below. Figure 4 shows $\mathrm{O}$ v $629 \AA$ data (row 1) along with EUVI $304 \AA$ images (row 2), SOT FG magnetograms (row 3), SOT Ca II H (row 4), EUVI $171 \AA$ (row 5) and XRT Al_poly images (row 6), taken over the course of the blinker event. The magnetic field configuration associated with this event is marked with a white box (Fig. 4, rows 3 and 4). Figure 5 shows the corresponding light-curves which have similar intensity enhancements in $\mathrm{Ca}$ II $\mathrm{H}$ (thin grey line), CDS O v $629 \AA$ (thick black line), EUVI $171 \AA$ (thin black line) and XRT Al_poly (thick grey line) with a lifetime of $50 \mathrm{~min}$, $43 \mathrm{~min}, 12 \mathrm{~min}$ and $8 \mathrm{~min}$ in the respective wavelengths. Figure 5 also shows the light-curves of the associated positive (dotted line) and negative (dashed line) magnetic fluxes (Fig. 4, row 3 ).

The blinker activity started with the emergence of bipolar magnetic fluxes in the internetwork region as observed in the magnetograms. The peak intensity occurred around 04:23 UT in CDS O v $629 \AA$. During the course of the event the preexisting negative polarity flux along with the emergence of weak positive polarity fluxes (Fig. 4, row 3, white box), resulted in a complete cancellation of the weak positive polarities. SOT $\mathrm{Ca}$ II $\mathrm{H}$ images (Fig 4, row 4) clearly show point-like (within the spatial resolution of the instrument, which is close to $100 \mathrm{~km}$ )
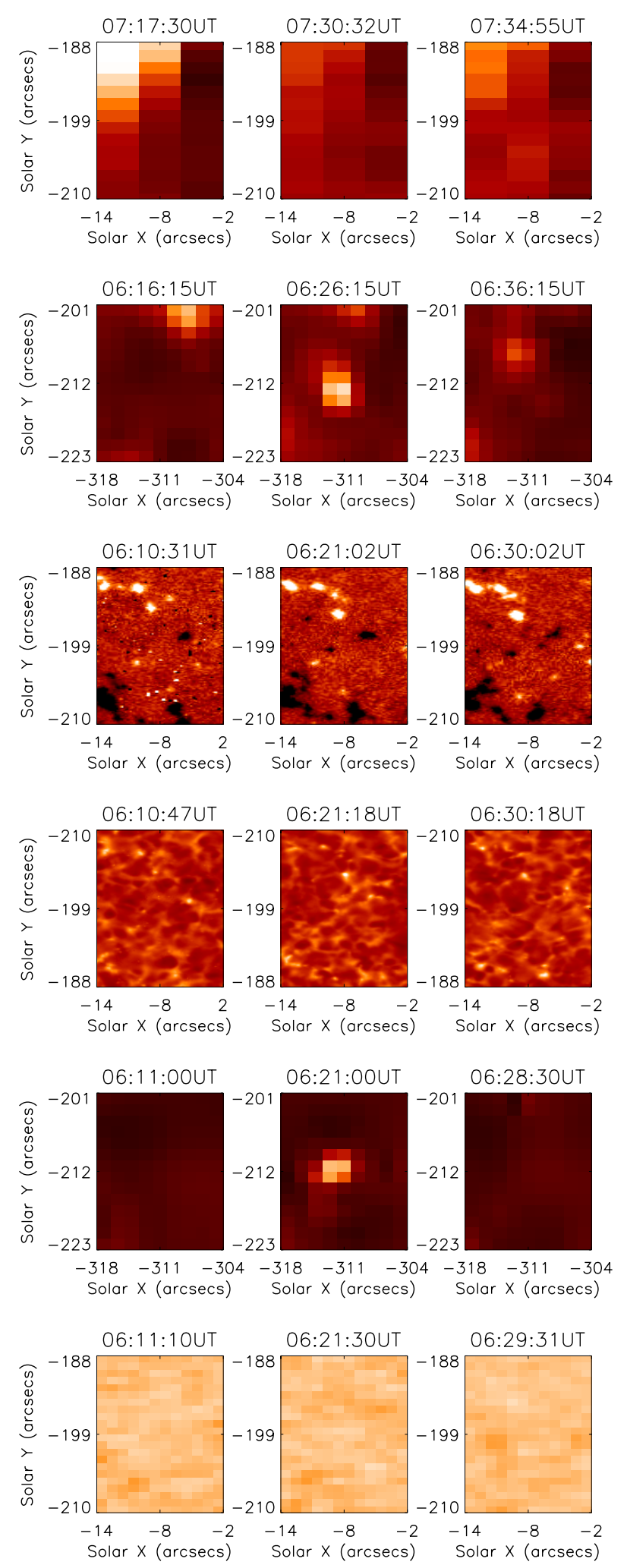

Fig. 6. From top, blinker group 3 in CDS O v $629 \AA$ A, EUVI $304 \AA$, SOT FG magnetograms, SOT Ca II H, EUVI 171 A and XRT Al_poly.

brightening activity at chromospheric heights which corresponds to the strong negative polarity flux. The event is also observed at coronal heights in the EUVI $171 \AA$ (lower corona) and in the X-ray Al_poly images as seen in Fig. 4 (rows 5 and 6). The X-ray images show that loop structures were formed around the peak 
of the blinker activity. No plasma ejections were observed in this event at coronal heights. We can only speculate that the cancellation of the bipolar fluxes triggered the brightening of a preexisting loop structure. The loop structure disappeared with the blinker event.

Though we see BG2 prominently in the EUVI and X-ray data, it was not identified by the identification procedure. As BG2 was followed by the formation of a coronal bright point, the corresponding light curves were dominated by this bright point (Fig. 5) at coronal temperatures (EUVI $171 \AA$ and X-rays). This caused an estimation of a very high local background and corresponding brightening identification threshold. Consequently, the BG2 which has a weaker radiance with respect to the bright point's radiance but a clear presence at coronal temperatures, was not identified by the automatic procedure. This example shows the limitations of our automated identification procedure which needs to be coupled with additional visual inspection in cases such as BG2.

During BG2 another positive polarity flux emerged (Fig. 4, row 3, dotted box) which moved away from the negative polarity flux discussed above (Fig. 4, row 3, solid box), resulting in the formation of a coronal bright point. Once the coronal bright point was formed at TR temperatures, it rose quickly to higher coronal temperatures (X-rays), producing a coronal jet in this region at around 05:15 UT with a corresponding blinker activity. The bright point produced two more coronal jets/blinkers over the course of our observations. The bright point also showed another 6 blinker events with no coronal jets. As coronal bright points can have a range of lifetimes from 8 to $50 \mathrm{~h}$ or longer (Golub et al. 1974; Ugarte-Urra et al. 2004), they are likely to produce several jets and blinkers over their lifetime. This blinker/jet falls under the category of events associated with a coronal bright point detected at X-ray temperatures (Subramanian et al. 2010).

\subsection{Blinkers with no X-ray counterparts}

The twelve remaining blinker groups, with a lifetime of around 20 min in CDS O v $629 \AA$, showed no detectable counterparts in the XRT images. One such group is discussed below, referred to as blinker group 3 (BG3).

Figure 6 shows BG3, consisting of two identified brightenings observed in CDS O v $629 \AA$ (row 1), EUVI $304 \AA$ (row 2) and EUVI $171 \AA$ (row 5), while the XRT Al_poly (row 6) showed no corresponding activity. The SOT FG magnetograms do not show any prominent changes of the magnetic fluxes (row 3 ) over the course of the event and no corresponding brightening could be detected in the $\mathrm{Ca}$ II $\mathrm{H}$ data (row 4). This strongly suggests that the energy deposition occurred at TR heights. Figure 7 also shows the light-curves of BG3 in CDS O v $629 \AA$ (thick black line), EUVI $171 \AA$ (thin black line), SOT Ca II H (thin grey line) and XRT Al_poly (thick grey line). The lightcurves can be seen to peak simultaneously in CDS O v $629 \AA$ and EUVI $171 \AA$. The lifetime of BG3 is 26 min in CDS O v $629 \AA$, while it is 13 min in EUVI $171 \AA$. This type of blinker group shows a brightening with no visible plasma ejection at coronal heights.

\subsection{Blinkers in EIS}

From the above results, the question remains as to whether the observed EUVI and X-ray blinker counterparts are real coronal

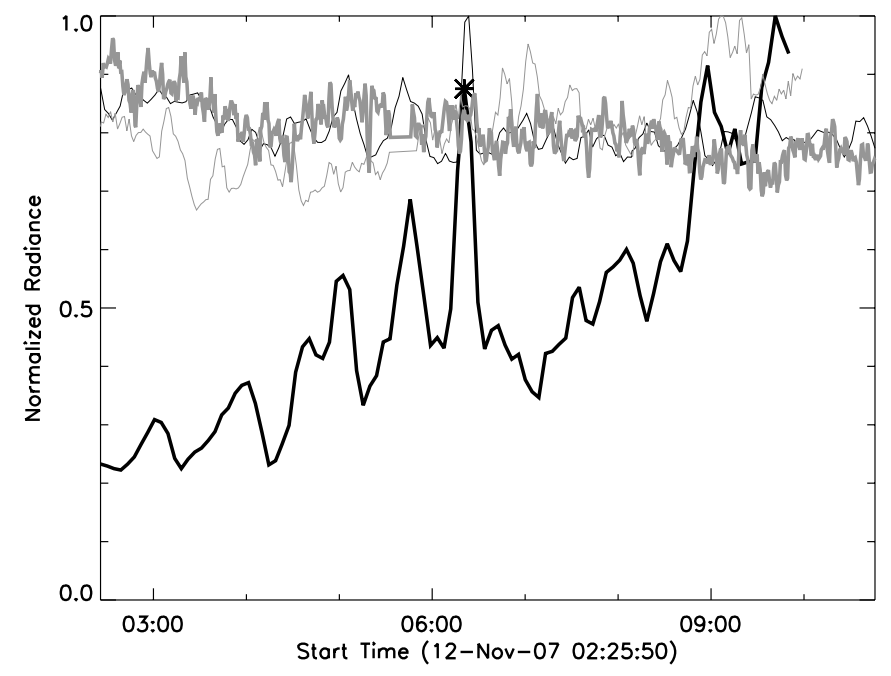

Fig. 7. Light-curves during blinker group 3 in CDS O v $629 \AA$ (thick black line), EUVI $171 \AA$ (thin black line), SOT Ca II H (thin grey line) and XRT Al_poly (thick grey line). The asterisk symbol indicates the peak in CDS O v $629 \AA$.

counterparts of blinkers or not. Both the EUVI $171 \AA$ and the XRT Al_poly filters have a wide temperature response including TR temperatures (around $\log T=5.5 \mathrm{~K}$ ). This motivated us to investigate blinkers further using spectroscopic data from Hinode/EIS which is currently the best spectrometer for studying coronal features. We could not study the brightening events discussed above with EIS, as the corresponding CDS data did not have an overlap with the EIS field-of-view. Hence, we used only the large raster from EIS which overlaps with the CDS large raster. Our study has clearly indicated so far that any blinker found in CDS O v $629.62 \AA$ has a counterpart in EUVI $171 \AA$. Therefore, brightening events identified in the coronal hole in EUVI $171 \AA$ data were used (Fig. 8) to study blinker counterparts in the EIS observations. Figure 8 shows the intensity maps of the large EIS raster along with the EUVI $171 \AA$ and XRT image of the same region. We detected 22 brightening events in the EUVI $171 \AA$ images that correspond to the field-of-view and timing of the large EIS raster. Out of the 22 brightening events, EIS rastered 12 of them over the course of the raster and all 12 events were clearly observed in the EIS O v $192.90 \AA$ rasters. Similar to SDO/AIA $171 \AA$ filter studied recently by Del Zanna et al. (2011), the EUVI $171 \AA$ filter has strong contribution from TR emission which is coming from $\mathrm{O} v$ and vi lines along with Fe IX which is sensitive to very cool, down to $\log T=5.5 \mathrm{~K}$ emission. Single Gaussian line fit was applied to the EIS data to derive the intensity map, except for the O v $192.90 \AA$ line which is blended in the blue wing by an Fe XI line. The two lines were separated by applying a double Gaussian line fit. Comparison of the CDS O v $629 \AA$ and EIS O v $192.90 \AA$ rasters (Fig. 9) clearly shows that we successfully subtracted the Fe XI contribution.

The coronal hole is indistinguishable in the O v $192.90 \AA$ line $\left(\log T_{\max }=5.4 \mathrm{~K}\right)$, while the Fe VIII $185.21 \AA \operatorname{line}\left(\log T_{\max }=\right.$ $5.8 \mathrm{~K}$ ) shows a trace of the coronal hole. The coronal hole is clearly visible in the Fe x $184.590 \AA$ and Fe xII $195.12 \AA$ coronal lines. The He II $256 \AA$ line is the spectral line with the lowest formation temperature available in the EIS spectral range. However, the interpretation of this line is complicated by blends with Si x 256.37 $\AA$, Fe xIII 256.42 $\AA$ and Fe xII 256.41 

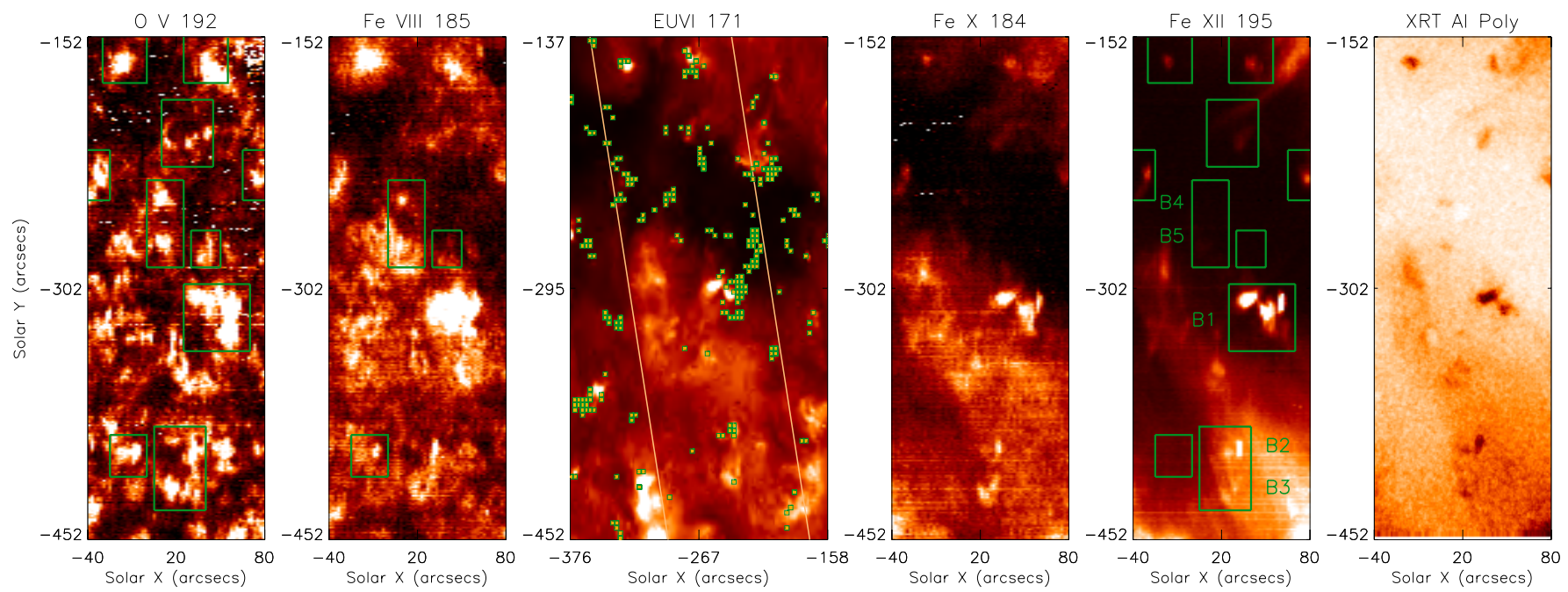

Fig. 8. EIS rasters of an ECH region from 2007 November 12. From left, EIS O v $192.90 \AA$ and Fe vIII $185.21 \AA$ intensity plots, EUVI $171 \AA$ image over-plotted with the brightenings identified as green boxes, EIS Fe x $184.590 \AA$ and Fe xII $195.12 \AA$ intensity plots, XRT Al_poly image. Box symbols over-plotted on the O v $629 \AA$ and Fe xII $195.12 \AA$ rasters show the 12 EUVI $171 \AA$ brightening events, with and without coronal counterparts at Fe XII temperatures. The boxes over-plotted on the Fe VIII 185.21 raster mark events with no coronal counterpart in Fe xII 195.12.
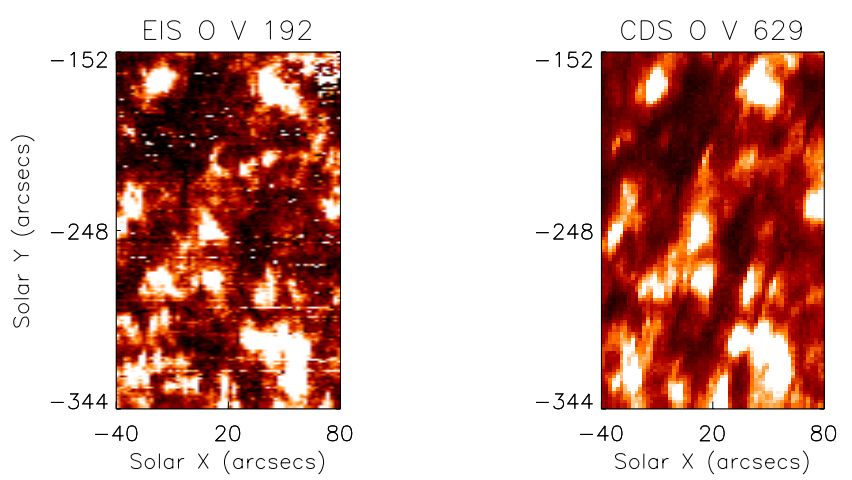

Fig. 9. From left: EIS large raster in O v $192.90 \AA ̊$ having common a FOV with the CDS large raster in O v $629 \AA$ A taken approximately at the same time.

\subsection{Blinkers with EIS Fe xII counterparts}

Out of the 12 EUVI $171 \AA$ brightenings observed by EIS, 4 of them showed counterparts in the EIS Fe VIII $185.21 \AA$, Fe x $184.590 \AA$ and Fe XII 195.12 $\AA$ lines and were associated with an X-ray jet. An example event, B1, is discussed below. Figure 10 (left) shows a sequence of XRT Al_poly and EUVI $171 \AA$ images during the event. The images show a coronal bright point and a coronal jet along with the disappearance of part of the bright point. This bright point persisted throughout the 10 hours of XRT Al_poly observations, producing multiple coronal jets.

Figure 10 (bottom) shows the light-curves of the blinker event B1 in XRT and EUVI $171 \AA$ A. This event falls under the category of a blinker associated with an X-ray coronal bright point jet.

The brightening B2, which is marked in Fig. 8, is another example of a blinker event with an X-ray jet. Fig. 11 (right) shows the event as seen in X-rays and EUVI $171 \AA$ A. Figure 11 (bottom) shows the light-curves of the jet peaking simultaneously in X-rays (thick line) and EUVI Fe $171 \AA$ (thin line). This event falls under the category of blinkers with an X-ray jet, but lacking a pre-existing coronal bright point in X-rays.

Four more events showed counterparts in EIS Fe VIII 185.21 $\AA$, Fe x $184.590 \AA$ and Fe XII $195.12 \AA$ with no observable plasma ejection in X-rays. An example event, B3, is discussed below. Plasma ejection associated with the blinker event/X-ray jet B2 (discussed above) triggered brightenings in the nearby loop structures giving rise to B3, as seen in the EUVI $171 \AA$ images, which then rose to higher coronal temperatures $(\log T=6.0 \mathrm{~K}$ and above). The blinker event B3 as seen in the EIS O v $192.90 \AA$ is probably the brightening of TR loops and/or their footpoint brightening. This event belongs to the category of blinkers associated with unresolved X-ray brightenings as discussed by Subramanian et al. (2010).

\subsection{Blinkers with no EIS Fe XII counterparts}

The remaining 4 events showed no counterpart to the blinker in the EIS Fe x $184.590 \AA$ and Fe XII 195.12 A lines. Although one event was identified in X-rays, the remaining 3 events did not have a counterpart in X-rays. Two events showed corresponding point-like brightening in the EIS Fe VII $185.21 \AA$ intensity maps (see Fig. 8), while the other two showed only a dispersed brightening in that area.

A sample brightening event B4, which is marked in Fig. 8, is discussed below. The event is clearly distinguishable in the EIS O v $192.90 \AA$ line. In XRT data, this event falls under the category of unresolved brightening with no plasma ejection. While in the EUVI $171 \AA$ images (Fig. 12), a point-like brightening (within the spatial resolution of the instrument, which is a few arcseconds) was observed with a corresponding formation/brightening of a loop structure, giving rise to another brightening B5 at the other end of the observed loop structure. The brightening B5 itself is a small loop structure in the EUVI $171 \AA$ images and it was not identified in X-rays. In the EIS Fe VIII $185.21 \AA$ line, brightening B4 showed a point-like brightening (again, within the spatial resolution of the instrument, similar to EUVI), while B5 could not be identified. The 

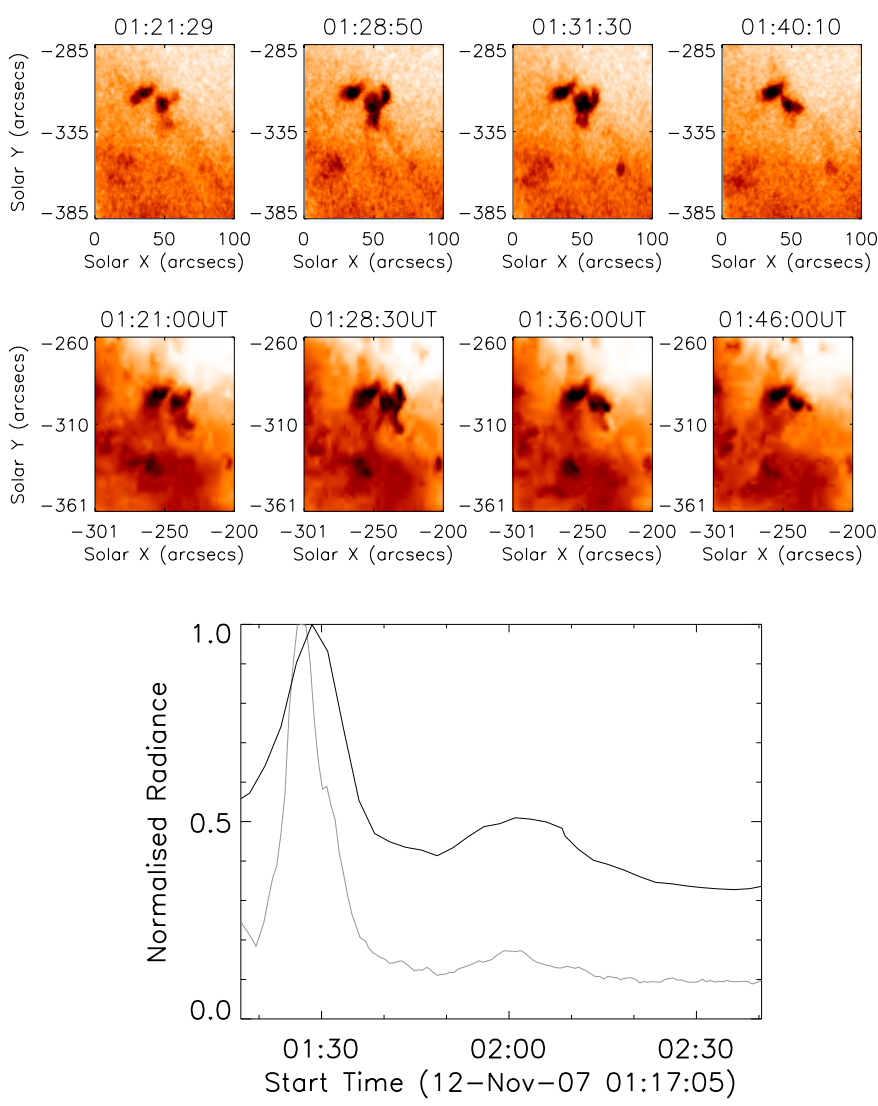

Fig. 10. The coronal bright point jet B1 Fig. 8) showing expansion and eruption of loop structures as observed in XRT Al_poly and EUVI $171 \AA$ images. The bottom panel shows the light-curves of the blinker event B1 peaking simultaneously in XRT (grey line) and EUVI $171 \AA$ (black line).

B5 appearance in the EUVI $171 \AA$ pass-band is, therefore, due to the contribution of TR emission as discussed earlier (Sect. 4.3). Figure 12 shows the light-curve of the brightening B4 in X-rays and EUVI 171 A.

\section{Discussion and conclusions}

The aim of the present study was to identify the true nature of the transient EUV brightenings, called blinkers, or, in other words, to find what causes the observed transient brightenings. We also investigated whether they have coronal signature, i.e., the link between EUV and coronal transient features, thereby the contribution of events associated with blinkers to coronal heating.

Twenty eight blinker groups were identified in CDS O v $629 \AA$ raster images. All of them showed a counterpart in EUVI $171 \AA$ and $304 \AA$ images and 57\% of them showed counterpart in X-rays. Although, blinkers were seen in EUVI $171 \AA$ and X-ray images, the question on whether they can reach coronal temperatures remained open, due to the significant contribution of transition-region emission to the $171 \AA$ pass-band and X-ray Al poly filters. A set of 12 blinker groups automatically identified in EUVI $171 \AA$ were studied with EIS in order to determine whether these brightenings have a coronal counterpart. Out of the 12 blinker groups, 8 were found to have an EIS Fe XII 195.12 ̊ counterpart suggesting that events
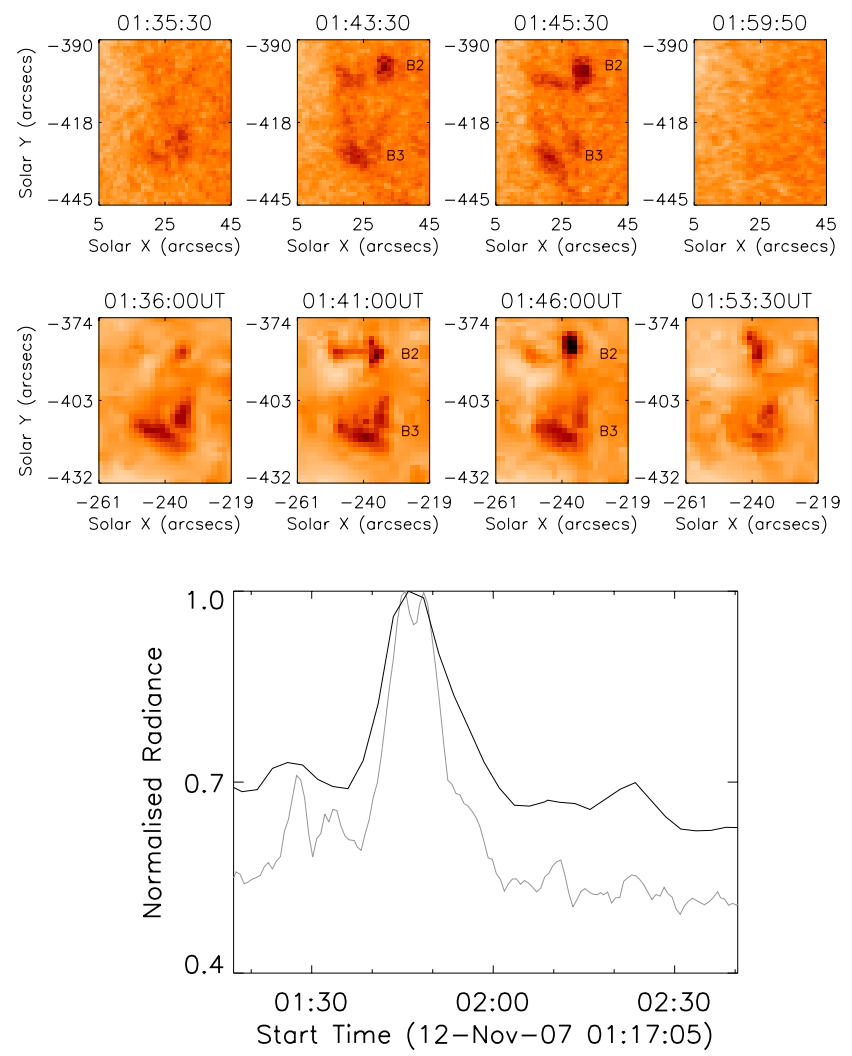

Fig. 11. The brightening B2 associated coronal jet and no pre-existing coronal feature (Fig. 8) showing plasma ejection as observed in XRT Al_poly and EUVI $171 \AA$ images. The bottom panel shows the lightcurves of the blinker event B2 peaking simultaneously in XRT (grey line) and EUVI $171 \AA$ (black line).

associated with the blinkers can release thermal energy directly in the solar corona. We found, in general, that blinkers are associated with various coronal events, e.g. EUV/X-ray jets, brightenings in small-scale loops (coronal bright points) or foot-point brightenings of larger loops.

Brightening events having no counterparts in X-rays and/or in EIS Fe XII 195.12 A were also studied. They appear as pointlike brightenings in CDS O v $\AA$ and EUVI $171 \AA$ images. As they do not reach coronal temperatures $(\geq \log T=6.0 \mathrm{~K})$, they certainly are associated with chromospheric features, e.g. larger spicules (O'Shea et al. 2005; Madjarska et al. 2006, 2011; Sterling et al. 2010) or have transition region origin, e.g. flows in small scale TR loops (Teriaca et al. 2004; Subramanian et al. 2008). Even these blinkers reach lower coronal heights (upto $\log T=5.8 \mathrm{~K}$ ). We found that some blinkers were clearly associated with newly emerging magnetic flux which caused the formation of loop structures seen in EUVI $171 \AA$ and X-ray images. Both the loops and the blinkers faded away as the bipolar fluxes drifted away from each other similar to the quiet Sun blinker event studied by Subramanian et al. (2008), thereby confirming this to be one of the mechanisms responsible for blinkers.

Blinkers with coronal counterpart occur repetitively and have a lifetime of around $40 \mathrm{~min}$ at transition region temperatures, while blinkers with no coronal counterpart, i.e. with transition region/chromosphere origin, happen only once and have a duration of $20 \mathrm{~min}$ in average. In general, lifetimes of blinkers are different at different wavelengths, i.e. different temperatures, decreasing from the chromosphere to the corona. 

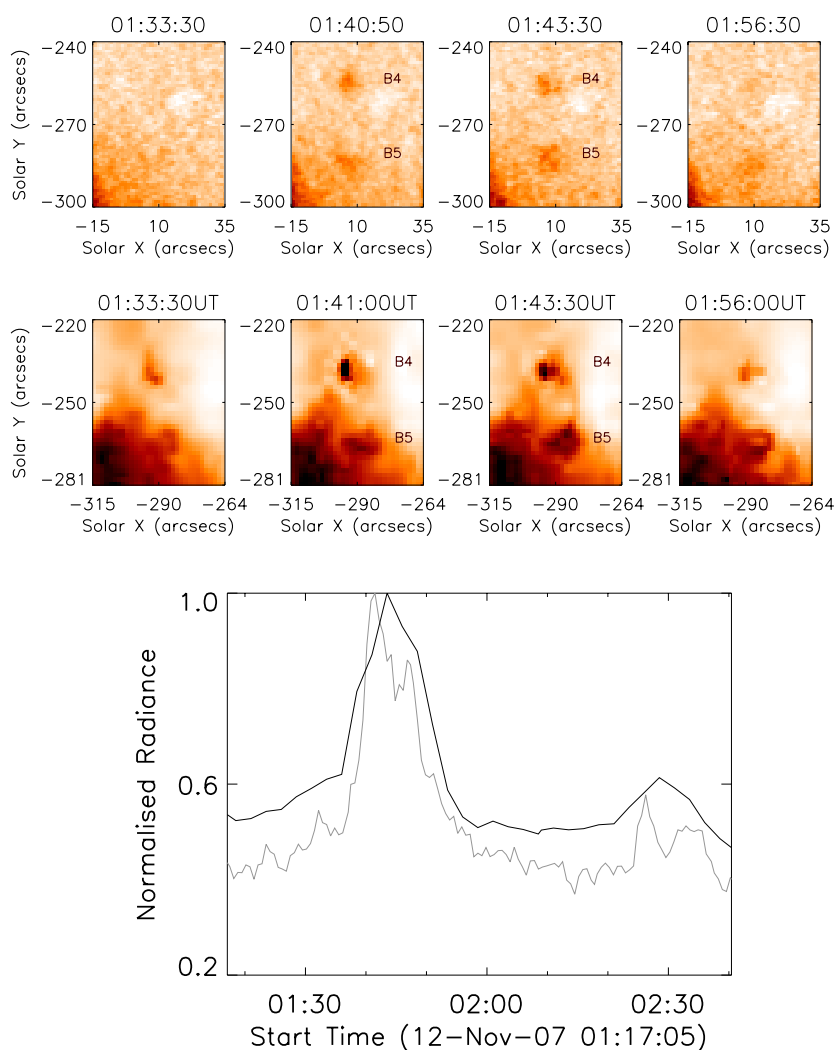

Fig. 12. Brightening events B4 and B5 (Fig. 8) with no coronal counterparts in Fe XII 195.12 as observed in XRT Al_poly (top) and EUVI $171 \AA$ (bottom) images. The bottom panel shows the light-curve of the brightening in X-rays and EUVI $171 \AA$.

The above results show that the term blinker is a "catchall" term for a vast range of transient events of coronal, transition-region or chromospheric origin. We find that the blinkers with coronal counterpart $(\geq \log T=6.0 \mathrm{~K})$ are associated with coronal activities and the blinkers with no counterpart above $\log T=6.0 \mathrm{~K}$ are associated with chromospheric features. Thus, up-flows from the chromosphere and down-flows from the corona associated with these blinkers do contribute to the formation and maintenance of the temperature gradient in the transition region.

Acknowledgements. The authors thank ISSI, Bern for the support of the team "Small-scale transient phenomena and their contribution to coronal heating". Research at Armagh Observatory is grant-aided by the N. Ireland Department of Culture, Arts and Leisure. We also thank STFC for support via grant ST/F001843/1. Hinode is a Japanese mission developed and launched by ISAS/JAXA, with NAOJ as domestic partner and NASA and STFC (UK) as international partners. It is operated by these agencies in co-operation with ESA and NSC (Norway). The STEREO project is an international consortium led by the Naval Research Laboratory (USA), including the Lockheed Martin Solar and Astrophysics Laboratory (USA), the NASA Goddard Space Flight Center (USA), the Rutherford Appleton Laboratory (UK), the University of Birmingham (UK), the Max-Planck-Institut für Sonnensystemforschung (Germany), the Centre Spatial de Liège (Belgium), the Institut d'Optique Thèorique et Appliquée (France) and the Institut d'Astrophysique Spatiale (France).

\section{References}

Bewsher, D., Parnell, C. E., \& Harrison, R. A. 2002, Sol. Phys., 206, 21 Bewsher, D., Parnell, C. E., Pike, C. D., \& Harrison, R. A. 2003, Sol. Phys., 215, 217

Brković, A., \& Peter, H. 2003, A\&A, 406, 363

Brković, A., Solanki, S. K., \& Rüedi, I. 2001, A\&A, 373, 1056

Brooks, D. H., \& Kurokawa, H. 2004, ApJ, 611, 1125

Brooks, D. H., Kurokawa, H., Kamio, S., et al. 2004, ApJ, 602, 1051

Culhane, J. L., Harra, L. K., Doschek, G. A., et al. 2005, Adv. Space Res., 36, 1494

Culhane, J. L., Harra, L. K., James, A. M., et al. 2007, Sol. Phys., 243, 19

Del Zanna, G., O’Dwyer, B., \& Mason, H. E. 2011, A\&A, 535, A46

Filippov, B., Golub, L., \& Koutchmy, S. 2009, Sol. Phys., 254, 259

Golub, L., Krieger, A. S., Silk, J. K., Timothy, A. F., \& Vaiana, G. S. 1974, ApJ, 189, L93

Golub, L., Deluca, E., Austin, G., et al. 2007, Sol. Phys., 243, 63

Harrison, R. A. 1997, Sol. Phys., 175, 467

Harrison, R. A., Sawyer, E. C., Carter, M. K., et al. 1995, Sol. Phys., 162, 233

Harrison, R. A., Lang, J., Brooks, D. H., \& Innes, D. E. 1999, A\&A, 351, 1115

Howard, R. A., Moses, J. D., Vourlidas, A., et al. 2008, Space Sci. Rev., 136, 67

Madjarska, M. S. 2011, A\&A, 526, A19

Madjarska, M. S., Doyle, J. G., Hochedez, J.-F., \& Theissen, A. 2006, A\&A, 452, L11

Madjarska, M. S., Vanninathan, K., \& Doyle, J. G. 2011, A\&A, 532, L1

Moore, R. L., Falconer, D. A., Porter, J. G., \& Suess, S. T. 1999, ApJ, 526, 505

O'Shea, E., Banerjee, D., \& Doyle, J. G. 2005, A\&A, 436, L43

Parker, E. N. 1988, ApJ, 330, 474

Parnell, C. E., Bewsher, D., \& Harrison, R. A. 2002, Sol. Phys., 206, 249

Priest, E. R., Hood, A. W., \& Bewsher, D. 2002, Sol. Phys., 205, 249

Schrijver, C. J., Title, A. M., van Ballegooijen, A. A., Hagenaar, H. J., \& Shine, R. A. 1997, ApJ, 487, 424

Schrijver, C. J., Title, A. M., Harvey, K. L., et al. 1998, Nature, 394, 152

Shibata, K., Ishido, Y., Acton, L. W., et al. 1992, PASJ, 44, L173

Shibata, K., Nitta, N., Matsumoto, R., et al. 1994, in X-ray solar physics from Yohkoh, ed. Y. Uchida, T. Watanabe, K. Shibata, \& H. S. Hudson, 29

Shimojo, M., Narukage, N., Kano, R., et al. 2007, PASJ, 59, 745

Sterling, A. C., Moore, R. L., \& DeForest, C. E. 2010, ApJ, 714, L1

Subramanian, S., Madjarska, M. S., Maclean, R. C., Doyle, J. G., \& Bewsher, D. 2008, A\&A, 488, 323

Subramanian, S., Madjarska, M. S., \& Doyle, J. G. 2010, A\&A, 516, A50

Teriaca, L., Banerjee, D., Falchi, A., Doyle, J. G., \& Madjarska, M. S. 2004, A\&A, 427, 1065

Tsuneta, S., Ichimoto, K., Katsukawa, Y., et al. 2008, Sol. Phys., 249, 167

Ugarte-Urra, I., Doyle, J. G., Madjarska, M. S., \& O'Shea, E. 2004, A\&A, 418, 313

Walsh, R. W., Ireland, J., Harrison, R. A., \& Priest, E. R. 1997, in Fifth SOHO Workshop: The Corona and Solar Wind Near Minimum Activity, ed. A. Wilson, ESA Special Publication, 404, 717

Winebarger, A. R., Emslie, A. G., Mariska, J. T., \& Warren, H. P. 2002, ApJ, 565, 1298

Yamauchi, Y., Wang, H., Jiang, Y., Schwadron, N., \& Moore, R. L. 2005, ApJ, 629,572

Yokoyama, T., \& Shibata, K. 1995, Nature, 375, 42 